Chirurg 2020 $91: 166$

https://doi.org/10.1007/s00104-020-01127-5

Online publiziert: 28. Januar 2020

(c) Springer Medizin Verlag GmbH, ein Teil von Springer Nature 2020

\section{Originalpublikation}

Al-Batran SE, Homan M, Pauligk C et al (2019) Perioperative chemotherapy with fluouracil plus leucoverin, oxaliplatin, versus doxetacel versus fluouracil, or capecitabine plus cisplatin and epirubicin for locally advanced, resectable gastric or gastro-oesophageal junction adenocarcinoma (FLOT4): a randomized phase $2 / 3$ trial. Lancet 393:1948-1957

Hintergrund und Fragestellung. In der S3-Leitlinie „Magenkarzinom“ ist die perioperative Chemotherapie als Standard für die lokal fortgeschrittenen Magenkarzinome und Karzinome des gastroösophagealen Übergangs Typ II und III definiert. $\mathrm{Zu}$ dieser Fragestellung wurden vor 2 Jahren von der AIO (Arbeitsgemeinschaft Internistische Onkologie) die Kurzzeitergebnisse einer prospektiv-randomisierten, multizentrischen Studie vorgestellt, in welcher die beiden gegenwärtig praktizierten Schemata (FLOT: 5-Fluouracil[FU]/Leukoverin/ Oxaliplatin/Doxetacel vs. ECF: Epirubicin/Cisplatin/5-FU) verglichen wurden. Als wesentliches Ergebnis wurde für das FLOT-Protokoll eine signifikant höhere Rate eines histopatholgischen „complete response" publiziert (16\% vs. $6 \%$, $p=0,02)$. In der aktuellen Publikation wurden jetzt von der deutschen Arbeitsgruppe die Langzeitergebnisse mit Überlebensdaten vorgestellt.

Methoden. In dieser Phase-2/3-Studie wurden in 38 deutschen onkologi-

W. Schröder ${ }^{1}$ C. J. Bruns

'Klinik für Allgemein-, Viszeral- und Tumorchirurgie, Universitätsklinik Köln, Köln, Deutschland

\title{
Langzeitüberleben nach perioperativer Chemotherapie beim Karzinom des gastroösophagealen Übergangs und Magens - ECF versus FLOT
}

schen Zentren 716 Patienten mit einem lokal fortgeschritten Magenkarzinom oder Karzinom des gastroösophagealen Übergangs (AEG Typ I-III) randomisiert. Nach Studienprotokoll erhielten 356 Patienten prä- und postoperativ jeweils 4 Zyklen FLOT und 360 Patienten jeweils 3 Zyklen ECF. Primärer Endpunkt war das Gesamtüberleben, als sekundärer Endpunkt wurde die chemotherapieassoziierte Toxizität („serious adverse events“, SAEs) analysiert. In der „Intention-to-treat“-Analyse konnten insgesamt 341 Patienten in der ECFGruppe und 345 Patienten in der FLOTGruppe operiert werden. Postoperativ erhielten lediglich 187 Patienten der ECF-Gruppe (51,9\%) und 213 Patienten der FLOT-Gruppe $(59,8 \%) \geq 1$ Zyklus Chemotherapie.

Ergebnisse. Als wesentliches Ergebnis dieser Studie konnte gezeigt werden, dass das mediane Überleben der mittels FLOT behandelten Patienten signifikant länger war verglichen mit der ECF-Gruppe (50 Monate vs. 35 Monate; Hazard Ratio 0,77; $95 \%$-Konfidenzintervall[CI] $0,63-0,94, p=0,012)$. Dementsprechend war auch die berechnete 5-Jahres-Überlebensrate in der FLOT-Gruppe signifikant höher (45\% vs. $36 \%$ ). Auch wenn sich das Spektrum chemotherapieassoziierter Toxizität unterschied, war die Gesamtrate der SAEs in beiden Gruppen mit $27 \%$ gleich, ebenso wie die chemotherapieassoziierte Todesrate mit $<1 \%$. Die R0-Resektionsrate war mit $78 \%$ in der ECF-Gruppe und $85 \%$ in der FLOTGruppe auffallend niedrig. Chirurgische Morbidität und Mortalität war für beide Gruppen vergleichbar.

\section{Kommentar}

Die vorliegenden onkologischen Daten dieser konzeptionell guten Studie zeigen ein signifikant besseres Langzeitüberleben für Patienten, die nach dem FLOTProtokoll perioperativ therapiert wurden. Die S3-Leitlinie „Magenkarzinom“ hat diese Daten bereits in ihrer aktuellen Version vom letzten Jahr mit aufgenommen. Bei der relativ geringen Rate von Patienten, die auch postoperativ mit 4 Zyklen FLOT oder 3 Zyklen ECF chemotherapeutisch behandelt wurde, stellt sich die Frage nach dem onkologischen Nutzen der perioperativen im Vergleich zur einfachen neoadjuvanten Chemotherapie. Auch die noch ausstehende Subgruppenanalyse der gastroösophagealen Karzinome weiter differenziert nach Typ I-III, die gegenwärtig auch leitlinienkonform nach dem CROSS-Protokoll therapiert werden können, bleibt von Interesse.

\section{Korrespondenzadresse}

Prof. Dr. W. Schröder, FACS, FEBS

Klinik für Allgemein-, Viszeral- und

Tumorchirurgie, Universitätsklinik Köln Kerpener Str. 62, 50937 Köln, Deutschland wolfgang.schroeder@uni-koeln.de

Interessenkonflikt. W. Schröder und C. J. Bruns geben an, dass kein Interessenkonflikt besteht. 